\title{
Akutes Abdomen - eine interdisziplinäre Herausforderung
}

\section{Acute Abdomen - an Interdisciplinary Challenge}

\author{
Gesprächsleiter: W. Teichmann, Hamburg \\ Teilnehmer: R. Arbogast, Pforzheim \\ H.-F. Weiser, Rotenburg/Wümme
}

F. Hagenmüller, J.R. Izbicki, K. Rückert, Hamburg

\section{Einleitung}

Hinter der Diagnose «akutes Abdomen» können sich zahlreiche, in der Diagnostik und Therapie unterschiedliche Erkrankungen verbergen. Eine große Anzahl verschiedener Diagnostika, von der einfachen klinischen Untersuchung bis zur invasiven, hoch technisierten Bildgebungsdiagnostik, steht zur Verfügung. Um dieses Instrumentarium auch gerade in den heutigen Zeiten knapper Ressourcen und ökonomischer Zwänge sinnvoll zu nutzen, ist eine intensive interdisziplinäre Abstimmung der effizienten und sinnvollen diagnostischen und therapeutischen Schritte notwendig. Sehr häufig wird man in der täglichen Praxis mit der Frage konfrontiert, wie lange konservativ zu behandeln ist, wann interventionell und wann operiert werden sollte? Diese Fragen haben wir in unserem interdisziplinären Gespräch aufgeworfen. Sie werden von den eingeladenen Experten beantwortet.

\section{Frage: Wie beurteilen Sie den Stellenwert der Laparoskopie beim akuten Abdomen?}

Arbogast: Die Zahl der Mitteilungen über den Einsatz der Laparoskopie beim akuten Abdomen nimmt in den letzten 2-3 Jahren zu. Aus meiner Sicht muss man streng trennen zwischen der Laparoskopie beim akuten Abdomen als diagnostische Option und der Laparoskopie mit der Konsequenz, die Ursache des akuten Abdomens auf laparoskopischem Wege zu beherrschen. Aufgrund der in den meisten Fällen sicher zu stellenden Diagnose bleibt für den diagnostischen Einsatz der Laparoskopie nur wenig Raum. Sie ist im Sinne der therapeutischen Option jedoch nach unserer Erfahrung sinnvoll bei der akuten Appendizitis, der akuten Cholezystitis, beim Mesenterialinfarkt bzw. der Mesenterialvenenthrombose. Beim perforierten Ulcus duodeni hat die Laparoskopie nur innerhalb

der ersten Stunden danach einen Stellenwert, da zwar laparoskopisch die Ulkusübernähung leicht ausgeführt werden kann, jedoch die konsequenterweise durchzuführende Spülung der Bauchhöhle enorm zeitaufwendig bleibt und damit ein echter Vorteil für den Patienten nicht zu erzielen ist. Der Einsatz der Laparoskopie im Ileus mit oder ohne diffuse Peritonitis kann im Einzelfall diskutiert werden, jedoch ist das Risiko nach meiner Auffassung höher einzuschätzen als die Möglichkeit, laparoskopisch letztendlich die Ursache für den Ileus oder die Peritonitis auf laparoskopischem Wege beherrschen zu können. Bei der akuten Divertikulitis hat die Laparoskopie initial keinen Stellenwert, da zum günstigen Zeitpunkt dann die notwendige Kolonresektion entweder laparoskopisch oder konventionell erfolgen muss.

Zusammenfassend ist nach unserer Auffassung daher die Laparoskopie im Rahmen des akuten Abdomens kein zu empfehlender Standard, sondern sollte auf spezifische Krankheitsbilder oder bei auf anderem Wege nicht zu sichernder Diagnose als Option bestehen bleiben. Sicher wird man im Laufe der nächsten Jahre auf dem Boden fundierter Kenntnisse einen Katalog erarbeiten müssen, wann die Laparoskopie beim akuten Abdomen sinnvoll ist und für den Patienten eine Erleichterung bietet.

Hagenmüller: Für die Diagnostik des akuten Abdomens spielt die Laparoskopie keine Rolle, wohl aber für die Therapie, wenn es der vorausgegangenen Diagnostik gelungen ist, als Ursache des akuten Abdomens eine laparoskopisch therapierbare Erkrankung zu sichern. Am häufigsten wird die therapeutische Laparoskopie zum Einsatz kommen, wenn eine akute Cholezystitis zum akuten Abdomen geführt hat. Die Laparoskopie kann auch zum Ziel der Übernähung eines perforierten Ulcus ventriculi oder duodeni oder zur Sanierung einer Sigmadivertikulitis eingesetzt werden, wenngleich man hier mit einer hohen Rate an methodischem Umsteigen zu-

\begin{tabular}{ll}
\hline KARGER & @ 2002 S. Karger GmbH, Freiburg \\
Fax +4976145207 14 & Accessible online at: \\
$\begin{array}{l}\text { E-mail Information@Karger.de } \\
\text { www.karger.com }\end{array}$ & www.karger.com/journals/cga
\end{tabular}


gunsten der offenen Laparotomie rechnen muss, wenn ein akutes Abdomen vorliegt.

Izbicki: Bei einem jüngeren Patienten, der mit einem akuten Abdomen in die Klinik eingeliefert wird, besteht unseres Erachtens in folgenden Situationen eine sehr gute Indikation zur Notfalllaparoskopie: (1) Bei jungen Frauen mit akuten Unterbauchschmerzen zur Differentialdiagnostik gynäkologische Erkrankungen versus Appendektomie, (2) bei chronisch rezidivierenden Bauchschmerzen mit dem neu aufgetretenen Bild eines akuten Abdomens und (3) bei der sonographisch gesicherten akuten Cholezystitis als Ursache für ein akutes Abdomen stellt die Laparoskopie unseres Erachtens eine gute Indikation dar.

Anmerkung: Bei Fieber, erhöhten Entzündungsparametern und klinisch gegebener diffuser Abwehrspannung ohne klar zuordenbaren Beginn der Schmerzhaftigkeit sehen wir die Indikation zur CT-Untersuchung des Abdomens. Weiterhin ist ein gut eingespieltes laparoskopisches Team mit dem notwendigen Know-how für eine Notfalllaparoskopie eine Grundvoraussetzung für ein derartiges Vorgehen.

Rückert: Die diagnostische Laparoskopie (DL) beim akuten Abdomen stellt eine wertvolle Ergänzung der etablierten diagnostischen Methoden dar, die jedoch nur in definierten Fällen zum Einsatz kommt. Erst wenn aus Anamnese, klinischer Untersuchung - möglichst wiederholt durch denselben erfahrenen Untersucher - Labor und Ultraschall keine klare Entscheidung für oder gegen ein operatives Vorgehen resultiert, kann die DL ein wertvolles Hilfsmittel sein. Einen bedeutenden Stellenwert hat die DL bei der akuten Unterbauchperitonitis zur Klärung der Ursache und gegebenenfalls laparoskopischen Therapie, z.B. bei der Differentialdiagnose Appendizitis - Adnexitis und Divertikulitis. Selten ist beim Ileus, bei mesenterialen Durchblutungsstörungen oder bei Verdacht auf Perforationen im Magen-Darm-Trakt die DL erforderlich. Die Laparoskopie hilft im Einzelfall unnötige Laparotomien zu vermeiden und die rechtzeitige Laparotomie nicht unnötig zu verzögern. Sie steht am Ende der diagnostischen Prozeduren bei akutem Abdomen und sollte immer mit erwogen werden.

Weiser: Die Diagnose akutes Abdomen wird nach Anamnese und klinischer Untersuchung zumindest im Sinne der Arbeitsdiagnose gestellt. Entsprechend werden laborchemische und bildgebende Untersuchungen durchgeführt. Die Laparoskopie steht am Ende der diagnostischen Kaskade, wobei sich der Zeitpunkt ihres Einsatzes auch nach der vitalen Bedrohung des Patienten richten muss. Im Gegensatz zum unklaren Abdomen kommt in der eigenen Klinik die Laparoskopie bei vital gefährdeten Patienten praktisch nicht zum Einsatz. Die Indikation zur Laparoskopie wird eher selten gestellt; die Domäne dieses Verfahrens sehen wir in der Grauzone zwischen unklarem und akutem Befund. Insbesondere bei penetrieren- den Verletzungen der Bauchwand sowie bei kommunikationsunfähigen Patienten besteht die Hauptindikation. Der immer wieder diskutierte Einsatz der Laparoskopie im Rahmen intestinaler Durchblutungsstörungen ist aus eigener Erfahrung ebenfalls nicht oder nur in extremen Ausnahmefällen indiziert und aussagekräftig. Problematisch gestaltet sich hier die komplette Durchmusterung des Intestinums, sodass bei uns Laborchemie, Angiographie und gegebenenfalls diagnostische Laparotomie nach wie vor als die Verfahren der Wahl gelten.

Kommentar: Die Laparoskopie kommt bei den gesicherten Diagnosen Appendizitis und Cholezystitis zum Einsatz, da sie hier auch therapeutische Optionen bietet. Ein weiteres Indikationsgebiet wird übereinstimmend beim Vorliegen unklarer abdominaler Beschwerden gesehen sowie beim Vorliegen einer Unterbauchperitonitis bei jungen Frauen. Als Standardverfahren wird die diagnostische Laparoskopie beim akuten Abdomen nicht angewendet.

\section{Frage: Welche diagnostischen Maßnahmen halten Sie bei der Diagnose «akutes Abdomen» für sinnvoll und in welcher Reihenfolge sollten sie durchgeführt werden?}

Arbogast: Prinzipiell muss zwischen dem echten Notfall wie perforierende Abdominalverletzung bzw. intraabdominale Blutung, bei der für die präoperative Diagnostik bekanntermaßen wenig Zeit bleibt, und dem akuten Abdomen, bei dem eine sinnvolle, strategisch überlegte Diagnostik noch präoperativ möglich ist, unterschieden werden. Generell gilt immer noch der Satz von Hippokrates: «Beginnen muss man mit dem Wichtigsten und dem Augenfälligsten. Dazu ist es unumgänglich, alles zu erlernen, was man sehen, fühlen und hören, alles was man kennen und anwenden kann.»

Am Beginn jeder Diagnostik stehen die sorgfältige Anamnese und die körperliche Untersuchung, stets die Häufigkeit der Ursachen des akuten Abdomens berücksichtigend. Parallel hierzu kommt der gezielten Labordiagnostik eine nach wie vor große Bedeutung zu. Den größten Stellenwert müssen wir heute außerdem der Ultraschalldiagnostik in der Hand des Geübten zuschreiben. In über $80 \%$ der Fälle gelingt es, die zuvor klinisch vermutete Diagnose zu sichern. Auch Erkrankungen im Urogenitaltrakt, die ein akutes Abdomen auslösen können, sind mit dem Ultraschall in einem hohen Prozentsatz $\mathrm{zu}$ verifizieren. Nach wie vor kommt der Röntgendiagnostik ein hoher Stellenwert zu, wie Abdomenübersichtsaufnahme (freie Luft, Spiegelbildungen ) oder dem Kontrasteinlauf mit wasserlöslichem Kontrastmittel (Stenose durch Entzündung oder Tumor bzw. freie oder gedeckte Perforation bei Divertikulitis). Als nächstes wäre die CT einzusetzen, gerade bei den Fällen, bei denen die Ultraschalluntersuchung nicht hilfreich gewesen ist, und in Ausnahmefällen kann die abdominale Übersichtsangiographie weitere Informationen liefern. Die Indikation zur Punktionsdiagnostik ist heute nur noch in den 
seltensten Fällen gegeben. Bei unklaren Befunden erlangt der Einsatz der Laparoskopie zunehmende Bedeutung.

Bei Anwendung der unterschiedlichen radiologischen Diagnoseverfahren ist es unumgänglich, dass der Kliniker dem Radiologen die Informationen weitergibt, die bei der Diagnose im Vorfeld bereits gesichert sind. Es ist wenig zielgerichtet und hilfreich sowie aus ökonomischen Erwägungen abzulehnen, sofort mit einer CT oder gar Kernspintomographie des Abdomens die Untersuchung zu beginnen.

Hagenmüller:

1. Detaillierte Anamnese

2. Körperliche Untersuchung

3. Labordiagnostik

4. Ultraschalluntersuchung des Abdomens, des Retroperitoneums und der inneren Genitalorgane, Abdomen-Leeraufnahme

5. bei unzureichender Aussage der Ultraschalluntersuchung (z.B. wegen Luftüberlagerung) und unklarem klinischen Bild: CT des Abdomens

6. bei klinischem Verdacht auf ein penetrierendes Ulcus ventriculi oder duodeni: Ösophagogastroduodenoskopie, anschließend gegebenenfalls erneute Abdomen-Leeraufnahme mit der Frage nach Luft im Peritonealraum.

Im interdisziplinären Gespräch mit dem Chirurgen wird der Internist beim akuten Abdomen vor allem die Aufmerksamkeit auf internistische Begleitkrankheiten lenken, die zu einem akuten Abdomen führen können (z.B. Herzrhythmusstörungen und andere Emboliequellen beim klinischen Verdacht auf eine mesenteriale Embolie). Gelegentlich gelingt es dem Internisten, seinen chirurgischen Kollegen von einer Laparotomie abzuhalten, wenn aus der Vorgeschichte und dem klinischen Bild auf ein Mittelmeerfieber zu schließen ist. Meistens handelt es sich hierbei um Patienten aus dem Mittelmeerraum mit Bauchschmerzen, Fieber und multiplen abdominalen Voroperationen. In diesen Fällen ist der Nachweis einer Proteinurie häufig ein schnell verfügbarer diagnostischer Mosaikstein, weil bei diesen Patienten oft eine Amyloidose der Nieren vorliegt. Der Internist kann auch zur Reduktion der Operationsfreude seines chirurgischen Partners beitragen, wenn er das Krankheitsbild der intestinalen Pseudoobstruktion vermutet. Auch diese Diagnose stützt sich im Wesentlichen auf eine sorgfältige Anamneseerhebung.

Izbicki:

1. Klinische Untersuchung einschließlich rektal-digitaler Untersuchung, Auskultation

2. Laborwerte: Blutbild, CRP, Laktat, Retentionswerte, Amylase, Blutzucker, Gesamtbilirubin

3. Sonographie des Abdomens

4. Röntgen des Abdomens in zwei Ebenen

5. Urinstix-Untersuchung.

Nach Beendigung dieser Diagnostik sollte das weitere diagnostische Vorgehen differenziert und fallspezifisch erfolgen.
Beim Bild eines Dünndarmileus und bei einem erhöhten Laktatwert sehen wir die Indikation zur sofortigen Laparotomie bei Verdacht auf Mesenterialischämie (die Angiographie ist unseres Erachtens nicht mehr zeitgemäß).

Die Gastrografinpassage erscheint uns nur noch gelegentlich bei Patienten mit dem radiologischen Bild eines Dünndarmileus nach Voroperation indiziert. Der Kolonkontrasteinlauf als Notfalldiagnostikum wurde inzwischen unseres Erachtens aufgrund der rascheren und praktikableren Durchführbarkeit sowie der Möglichkeit, mehrere Fragestellungen auf einmal zu beantworten, vom Hydro-CT abgelöst (bei der Frage Sigmadivertikulitis, Sigmaperforation). Endoskopische Diagnostik beim akuten Abdomen erachten wir für indiziert, wenn sich in der zuvor applizierten Magensonde die Zeichen einer frischen oder älteren stattgehabten Blutung finden; bei einer peranalen Blutung ist eine Koloskopie bei dem meist nicht adäquat vorbereiteten Kolon nicht sinnvoll. Um eine leicht behebbare Hämorrhoidalblutung diagnostizieren zu können, ist nach unserer Meinung im Notfall bei einer unteren gastrointestinalen Blutung eine Rektoskopie bzw. Proktoskopie ausreichend.

Rückert: Die Diagnose «akutes Abdomen» ist ein Sammelbegriff, der nicht exakt definiert ist. Übereinstimmend handelt es sich um eine plötzlich aufgetretene, lebensbedrohliche Baucherkrankung, deren Ursache rasch erkannt werden muss, damit die zunehmend lebensbedrohliche Situation rechtzeitig beseitigt wird. Neben der Peritonitis (lokal oder diffus) sind der Ileus, die intraabdominale Blutung oder die mesenteriale Ischämie die häufigsten Ursachen.

Folgende diagnostische Maßnahmen sind in der angegebenen Reihenfolge sinnvoll: sorgfältige standardisierte Anamnese, systematische klinische Untersuchung, möglichst wiederholt durch denselben erfahrenen Untersucher, parallel dazu Bestimmung von Basislabor- und gegebenenfalls Speziallaborwerten. Die Sonographie durch einen erfahrenen Untersucher hat einen hohen Stellenwert. Mit diesen diagnostischen Maßnahmen lassen sich meist die häufigsten Ursachen des akuten Abdomens feststellen: akute Appendizitis, akute Cholezystitis, Ileus, Urolithiasis, akute Divertikulitis und perforiertes Ulkus.

Bleibt die Situation unklar, folgen die Abdomenübersichtsaufnahme (2 Ebenen), der Kontrasteinlauf und/oder ein CT. Im Einzelfall schließt sich die Endoskopie an, um eine Perforation ohne freie Luft ausschließen zu können. Für ausgewählte Fälle ist dann die DL indiziert oder eine FarbduplexUntersuchung bzw. Angiographie zur Erkennung einer mesenterialen Ischämie. Grundsätzlich gilt, so rasch wie möglich unter Vermeidung unnötiger Untersuchungen zur Diagnose zu kommen. Die explorative Laparotomie wird damit zur Ausnahme, darf jedoch nicht in Vergessenheit geraten.

Weiser: Entsprechend den Ausführungen zur 1. Frage bedeutet die Diagnose «akutes Abdomen» immer vitale Gefähr- 
dung des Patienten mit entsprechendem Zeitdruck der Diagnosestellung. Nach wie vor entscheidend ist eine detaillierte Anamnese (Ulkus-, Gallensteinleiden, Abklärung von möglichen Emboliequellen, Divertikulose-/Divertikulitiszeichen, eventuell B-Symptomatik usw.). Parallel dazu erfolgt die detaillierte klinische Untersuchung (Vitalparameter, Schmerzcharakter, -lokalisation und -ausstrahlung, Peritonismus, Darmgeräusche, tastbare Raumforderungen usw.). Bereits anhand dieser von jedem erfahrenen Arzt vergleichsweise schnell durchzuführenden Untersuchungen lässt sich bei vital gefährdeten Patienten die Indikation zu weiterführenden Maßnahmen bzw. zur sofortigen Laparotomie stellen. Parallel zur Anamneseerhebung und klinischen Untersuchung erfolgt die Untersuchung relevanter Laborparameter (Entzündungsparameter, Transaminasen und Pankreaswerte, Nekrosemarker bzw. Ischämiezeichen, Retentionswerte und Gerinnungsparameter). Je nach Arbeitsdiagnose und klinischem Zustand des Patienten kommen nachfolgend bildgebende Verfahren zum Einsatz:

1. Röntgen-Abdomen als Leeraufnahme im Stehen bzw. in Linksseitenlage, gegebenenfalls folgend mit wasserlöslichem Kontrastmittel als Kolonkontrasteinlauf oder Magen-DarmPassage. Bei Nachweis entzündlicher Veränderungen, z.B. Divertikulitis zusätzlich CT zur Beurteilung der Ausdehnung des entzündlichen Prozesses, gegebenenfalls gedeckter Perforationen oder Abszessformationen.

2. Wenn bei intestinaler Luftüberlagerung möglich, Sonographie zum Nachweis freier Flüssigkeit, Raumforderung, Pendelperistaltik sowie größeren entzündlichen Tumoren oder Abszessformationen.

3. Angiographie bei laborchemischem und klinischen Verdacht einer intestinalen Ischämie.

4. Laparoskopie in Einzelfällen.

Kommentar: Bezüglich der Reihenfolge der diagnostischen Maßnahmen besteht Konsens darüber, dass die detaillierte Anamnese und genaue klinische Untersuchung die Weichen für den weiteren klinischen Verlauf des Patienten stellen. Im zweiten Schritt sollte dann die Untersuchung der Blutwerte erfolgen, sodann wird in den meisten Fällen die Sonographie bevorzugt eingesetzt. Erst wenn nach diesen Schritten die Diagnose weiterhin unklar bleibt, wird das abdominale CT oder Hydro-CT eingesetzt.

\section{Frage: Peritonitis: Wann wird geschlossen behandelt und wann im Konzept der Etappenlavage?}

Arbogast: Bei der Behandlung der Peritonitis haben die Ausführungen von Kirschner aus dem Jahr 1926 uneingeschränkt ihre Bedeutung. Im Zentrum steht die Herdsanierung mit der konsequenterweise durchzuführenden Reinigung der Bauchhöhle und der gezielten Drainagebehandlung. Weiterführende
Konzepte wie das offene Abdomen, die geschlossene kontinuierliche Spülung der Bauchhöhle, die dorsoventrale Lavage und die Etappen- oder programmierte Lavage haben ihre Bedeutung nur bei der diffusen 4-Quadranten-Peritonitis. Hierbei muss man klar festhalten, dass alle Techniken der Laparostomie das zentrale Ziel der Entlastung des abdominalen Kompartments verfolgen und erst in zweiter Linie kommt der regelmäßigen Lavage der Bauchhöhle ein größerer Stellenwert zu. Alle lokalisierten Formen der Peritonitis sind nicht in das Programm der Etappenlavage mit einzubeziehen, lediglich mit der Ausnahme eines persistierenden peritonitisbedingten lleus mit der Gefahr der Sepsis durch eine Translokation von Mediatoren. In diesen Fällen ist eine Dekompression des Darms, z.B. über die Dennis-Sonde oder wiederum durch eine passagere Entlastung des abdominalen Kompartments, durchzuführen.

Entschließt man sich bei einer zunächst lokalisierten Form der Peritonitis zu dem klassischen, von Kirschner angegebenen Verfahren, so ist jedoch stets darauf zu achten, wie sich der weitere Verlauf abzeichnet. Besteht nur der geringste Zweifel, dass die zunächst lokal gebliebene Peritonitis in eine diffuse Form übergeht, so ist sofort ein Verfahrenswechsel zum Etappenlavage-Prinzip durchzuführen, da jede Verzögerung in der definitiv notwendigen Therapie die Letalität gravierend ansteigen lässt.

Hagenmüller: Bei dieser Frage sollte sich der Internist tunlichst nicht einmischen.

Izbicki: Für die geschlossene Behandlung einer Peritonitis gibt es unserer Meinung nach folgende Indikation: Lediglich die lokalisierte Peritonitis sollte geschlossen behandelt werden; bei der diffusen Peritonitis kann nach Fokussanierung ein Versuch unternommen werden, die Etappenlavage zu umgehen, allerdings nicht bei einer drastischen Volumenzunahme des Darms oder bei einem drohenden Kompartmentsyndrom. Bei der kotigen Peritonitis empfiehlt es sich aber - sollte ein geschlossenes Vorgehen gewählt werden - zumindest eine Second-look-Operation durchzuführen. Ansonsten stellt das Konzept der Etappenlavage den Goldstandard der Peritonitisbehandlung dar.

Rückert: Diese Frage lässt sich nicht auf Grund von publizierten Studienergebnissen beantworten, da diese fehlen. Hier fließen die individuellen Erfahrungen des Viszeralchirurgen neben verschiedenen klinischen Kriterien in die Entscheidungsfindung zur Verfahrenswahl mit ein. Unabhängig vom Verfahren muss jederzeit flexibel entschieden werden, ob dieses beibehalten werden darf oder gegebenenfalls frühzeitig ein alternatives Procedere gewählt wird. Die überaus großzügige Indikation zur Etappenlavage und die zu lange Durchführung sind genauso schädlich für den Patienten wie die fortdauernde geschlossene Behandlung bei fehlender Besserung des septisch-toxischen Zustands des Patienten. Die Standard- 
therapie bei diffuser Peritonitis ist Konsens - unabhängig vom weiteren Behandlungskonzept. Von wesentlicher Bedeutung ist die auf den individuellen Patienten zugeschnittene Beherrschung des abdominalen Kompartmentsyndroms.

Unseres Erachtens sollen Patienten mit diffuser (4-Quadranten-)Peritonitis, deren Ursache im unteren Gastrointestinaltrakt liegt und die aufgrund der Sepsis bereits Organkomplikationen haben, eher der Etappenlavage zugeführt werden als Patienten mit lokoregionärer Peritonitis ohne Organkomplikationen. Eine ein- oder zweimal durchgeführte Etappenlavage ist unseres Erachtens für den Patienten von geringerem Nachteil als die unterlassene Etappenlavage.

Weiser: Im eigenen Krankengut kommt die geschlossene Peritonitisbehandlung nicht zur Anwendung. Wir bevorzugen seit nunmehr 14 Jahren die sofortige und endgültige Fokussanierung mit nachfolgender Etappenlavage.

Die Fokussanierung wird im eigenen Krankengut auch unter Inkaufnahme von Diskontinuitätsresektionen, wann immer möglich, erzwungen. Im Rahmen der Etappenlavage wird die Bauchhöhle mit 10-12 1 Kochsalzlösung, gegebenenfalls mit Lavasept, gespült. Der Bauchdeckenverschluss erfolgt passager nach jeder Lavage über tief durchgreifende U-Nähte, gegebenenfalls unter Verwendung von Reißverschlusssystemen.

Kommentar: Einigkeit besteht darüber, dass die kausale Behandlung der Peritonitis durch eine Sanierung des Fokus erfolgen muss. Gelingt dieses nicht durch die Standardtherapie (Kirschner, 1926) oder bestehen eine schwere 4-QuadrantenPeritonitis bzw. ein abdominales Kompartmentsyndrom, so sollte der Patient im Konzept der Etappenlavage behandelt werden.

\section{Frage: Behandlungsoptionen bei Morbus-Crohn- Abszessen: Wann konservativ? Wann interventionell? Wann operieren?}

Arbogast: Die Ausbildung von intraabdominalen Abszessen bei M. Crohn ist im Vergleich zur Colitis ulcerosa in einem sehr viel höheren Prozentsatz zu erwarten. Bei M. Crohn sind sie meist Folgen von Fisteln, die im Rahmen einer transmuralen Entzündung entstanden sind. Die Abszesse liegen am häufigsten zwischen Darm und Bauchwand oder im Retroperitoneum, so genannte enteroparietale Abszesse. Sehr viel seltener sind die größeren Schlingenabszesse oder Abszesse im Bereich des Mesenteriums.

Bei gut zugänglichen Abszessen ist eine sonographie- oder CT-gesteuerte Drainage indiziert. In etwa 20\% der Fälle kann auch mit einer Ausheilung ohne eine enterokutane Fistel gerechnet werden. Die Erfahrung zeigt jedoch, dass die meisten Patienten einen zweiten Eingriff mit Resektion des fisteltragenden Darmabschnitts benötigen. Die interventionelle Drainage verhindert oder behandelt jedoch eine bereits bestehen- de Sepsis, ermöglicht die weitere Diagnostik und gestattet insbesondere eine Verbesserung der Situation des betroffenen Patienten für eine dann notwendige elektive Operation. Anlage von Stomata zur Behandlung von Abszessen sind in unserer Klinik obsolet.

Bei perineal vorliegenden Abszessen und bekanntem $\mathrm{M}$. Crohn sollten nach unserer Auffassung diese Abszesse nicht nach den klassischen Kriterien von abszessbegleitenden Fisteln im perinealen Bereich operiert werden, sondern lediglich die Abszesse entlastet und dann entweder auf konservativem (soweit möglich) oder operativem Weg die Crohn-bedingte Ursache dieser perineal vorliegenden Abszesse beseitigt werden. Ein rein konservatives Vorgehen bei nachgewiesenen Crohn-bedingten Abszessen ist nur selten von Erfolg gekrönt, weshalb der Weg über eine mögliche Intervention zur definitiv operativen Sanierung gewählt werden sollte.

Hagenmüller: Die konservative Therapie kommt nur für sehr kleine Abszesse in Frage. Mit einer hohen Misserfolgsrate ist zu rechnen, zum einen von Seiten des natürlichen Verlaufs dieser Erkrankung und zum anderen wegen der oft bestehenden, medikamentös induzierten Immunsuppression. Die perkutane Punktion von Abszessen kann man zur temporären Entlastung bei Fieber und/oder septischem Krankheitsbild einsetzen. In diesen Fällen wird eine spätere offen chirurgische Sanierung notwendig sein.

Izbicki: Die konservative Therapie bei M.-Crohn-Abszessen ist sicher nicht von einem langfristigen Erfolg gekrönt und sollte daher nur Ausnahmefällen vorbehalten bleiben. Die interventionelle Behandlung dieser Abszesse kommt unseres Erachtens nur als temporäre Behandlungsoption in Frage, um eine akute Entzündungssituation zu verbessern und den Patienten zwischenzeitlich in einen besseren, nichtseptischen Allgemeinzustand zu bringen, der das weitere operative Vorgehen erleichtert. Prinzipiell sollten intraabdominale M.Crohn-Abszesse bis auf wenige Ausnahmen operativ behandelt werden.

Rückert: Abszesse im Becken oder im Abdomen entwickeln bis zu 30\% der Patienten mit einem M. Crohn. Abhängig vom klinischen Bild und der Abszesslokalisation sowie der Anamnese eventueller Voroperationen und aktueller medikamentöser Therapie wird das weitere Vorgehen festgelegt. Bei kleinen Abszessen ohne Organkomplikation und Ileus erfolgt die konservative Therapie. Bei Abszessen mit Sepsis und Organkomplikation, die sich in dem bildgebenden Verfahren als risikoarm punktionsfähig erweisen, wird perkutan drainiert und nach Abklingen der akuten Symptomatik wird die Indikation zum operativen Vorgehen neu evaluiert.

Häufig muss beim intraabdominalen Crohn-Abszess offen chirurgisch vorgegangen werden, da eine hohe Morbidität mit einer hohen Rezidivquote bei konservativem oder rein interventionellen Vorgehen einhergeht. Wenige ausgewählte Pa- 
tienten profitieren im Hinblick auf die Rezidivabszessquote und die Vermeidung weiterer chirurgischer Eingriffe im Verlauf von alleinigen interventionellen Maßnahmen.

Weiser: Etwa 12-28\% der Patienten mit M. Crohn entwickeln intraabdominale Abszesse. Eine freie Perforation bei M. Crohn tritt dagegen nur maximal in 1-2\% der Fälle auf. Meist handelt es sich um Phlegmonen des terminalen Ileums im Rahmen einer transmuralen Penetration von Fissuren. In aller Regel werden diese durch Darmschlingen oder Netz abgedeckt mit der Folge der Ausbildung einer Abszessformation. Kommt es zu einer internen Drainage der Abszesse, können konservative Maßnahmen diskutiert und die in der Regel unumgängliche Operation mit aufgeschobener Dringlichkeit durchgeführt werden.

In extremen Ausnahmefällen können interventionelle Drainagemaßnahmen erwogen werden. Für ein interventionelles Vorgehen kommen allerdings nur seltene abgedeckelte und isolierte extraintestinale Abszesse, z.B. im Bereich des M. psoas in Frage. Im eigenen Krankengut hat die interventionelle Drainage von Crohn-Abszessen keinen Stellenwert, da in aller Regel spätere Operationen aufgrund sich einstellender komplizierter Verläufe erforderlich werden bzw. der Entwicklung intestinokutaner Fisteln Vorschub geleistet wird.

Das Regelvorgehen bei Crohn-Abszessen stellt im eigenen Krankengut die operative Revision dar. Als absolute Operationsindikation betrachten wir Abszesse, die im Zusammenhang mit ileovesikalen oder enterogenitalen Fisteln oder im Rahmen eine obstruktiven Uropathie auftreten. Insbesondere die in bis zu 5\% der Patienten zu erwartende obstruktive Uropathie durch Kompression meist des rechten Ureters bei Ileozökalphlegmone oder Abszess stellt aus unserer Sicht eine absolute Operationsindikation dar. Ebenfalls operativ saniert werden perianale Abszesse bei Crohn-Befall des Rektums bzw. im Rahmen eines komplizierten perianalen M. Crohn.

Kommentar: Die Therapie der interenterischen bzw. enteroparietalen M.-Crohn-Abszesse, die bei 10-28\% der Patienten auftreten, sollte nach übereinstimmender Meinung operativ erfolgen. Interventionelle Verfahren kommen überwiegend temporär zum Einsatz, um den Allgemeinzustand und den Lokalbefund zu verbessern.

\section{Frage: Therapie des toxischen Megakolons}

Arbogast: Das toxische Megakolon als schwerwiegendste Komplikation einer fulminanten Kolitis, meistens der Colitis ulcerosa, nimmt in seiner Häufigkeit ab. Die Mortalität ist nach wie vor hoch, insbesondere dann, wenn durch eine zeitliche Verzögerung des Therapiebeginns eine spontane Perforation resultiert. Eine rein konservative Therapie ist nur über ein Zeitintervall von maximal 12-24 h erlaubt. Bei nicht an- sprechender Therapie ist die Indikation zur Operation zwingend gegeben. Nach unserer Auffassung stellt die subtotale Kolektomie mit Ileostomie und Hartmann-Situation bzw. Sigmoidostomie als Schleimfistel das Verfahren der Wahl dar. Außerdem erlaubt dieses operative Vorgehen auch eine spätere Wiederherstellung durch Pouch. Die Turnbull(Blowhole)Methode findet an unserer Klinik seit vielen Jahren keine Anwendung mehr. Bei der Operation eines toxischen Megakolons ist strengstens darauf zu achten, dass Perforationen des Dickdarms mit Austritt der «toxischen Brühe» vermieden werden müssen.

Hagenmüller: Es gibt kein generell akzeptiertes und auf jeden Einzelfall anwendbares Therapiekonzept für das toxische Megakolon. Bei diesem Krankheitsbild ist die enge Kooperation zwischen der gastroenterologischen Intensivmedizin und der Chirurgie besonders wichtig. Die konservative Behandlung beinhaltet die engmaschige Beobachtung der metabolischen Situation, der Sepsisparameter, die radiologische Suche nach Hinweisen auf eine Darmperforation, die Drainage des Intestinums mittels intestinaler Sonden und/oder wiederholter endoskopischer Darmgasabsaugung, die Breitspektrumantibiose und immunsuppressive Therapie. Oft kann die konservative Therapie die Wende zur Besserung einleiten. Wenn der Patient allerdings nicht innerhalb weniger Tage auf diese Bemühungen anspricht, steigt die Folgemorbidität stark an. Verschiedene Arbeitsgruppen empfehlen, dass beim Nichtansprechen der konservativen Therapie der Entschluss zur Kolektomie nach 24-72 h gefasst werden sollte. Die Kolektomie erfolgt, um der drohenden Kolonperforation zuvorzukommen, die mit einer Sterblichkeit von $80 \%$ einhergeht.

Izbicki: Laparotomie, subtotale Kolektomie und je nach $\mathrm{Zu}$ stand des Patienten entweder Resektion in Diskontinuität im Sinne der Anlage eines kurzen Hartmann-Stumpfes und eines Ileostomas. Bei Patienten in einem guten Allgemeinzustand empfehlen wir die subtotale Kolektomie und die Anlage einer Ileorektostomie.

Rückert: Die akute toxische Kolitis und Kolondilatation ist eine lebensbedrohliche Erkrankung. Charakteristisch sind nichtobstruktive Kolondilatation $(>6 \mathrm{~cm})$ und septisch-toxischer Schock (Fieber, Tachykardie, Leukozytose und/oder Dehydratation, Elektrolytentgleisung, Hypotension). Die Behandlung erfolgt primär interdisziplinär mit Gastroenterologen, Intensivmediziner und Viszeralchirurgen. Colitis ulcerosa, M. Crohn und die pseudomembranöse Kolitis sind die Hauptursachen. Nach initialem Ausschluss einer Perforation erfolgt eine aggressive intensivmedizinische Behandlung zur Reduktion der Kolondilatation, zur Herstellung der Homöostase und zur Beherrschung des septisch-toxischen Schocks. Indikationen zur Notfalloperation sind die freie Perforation, die massive Blutung, nicht beherrschbarer toxischer Schock und zunehmende Kolondilatation. Bleibt eine klinische Besserung 
unter aggressiver medikamentöser Therapie (inkl. Hydrocortison) innerhalb von 24-72 h aus, erfolgt die subtotale Kolektomie mit Ileostoma und Hartmann-Stumpf. Nach eingetretener Perforation steigt die Letalität auf das 5 fache an. Das korrekte Timing kann nur interdisziplinär durch den Gastroenterologen und Chirurgen erfolgen.

Weiser: Folgende Situationen bedingen eine sofortige Operation: Pneumoperitoneum, gramnegative Sepsis, massive Blutung und diffuse Peritonitis. In den seltenen Fällen eines operationsbedürftigen toxischen Megakolons (in den letzten 5 Jahren im eigenen Krankengut nicht beobachtet) würden wir eine subtotale Kolektomie mit Verschluss des distalen Stumpfes und Anlage eines endständigen Ileostomas vornehmen. Dieses Vorgehen hat eine geringere Letalität als die Proktokolektomie und erlaubt eine spätere exakte Differenzierung zwischen einem toxischen Megakolon bei M. Crohn oder Colitis ulcerosa, so dass der Weg für eine spätere Proktomukosektomie und Pouch-Bildung nicht versperrt wird.

Kommentar: Am Krankheitsbild des toxischen Megakolons wird die hohe Bedeutung enger interdisziplinärer Kommunikation deutlich. Ein konservativer Behandlungsversuch der heute seltenen Komplikation entzündlicher Darmerkrankungen ist auf 12 bis maximal $72 \mathrm{~h}$ zu begrenzen. Voraussetzung hierfür ist das Fehlen von Zeichen der freien Perforation bzw. einer generalisierten Peritonitis.

Bleibt nach dieser Zeitspanne eine Besserungstendenz aus, so besteht die Indikation zur subtotalen Kolektomie, die je nach Zustand des Patienten kontinuitätserhaltend mit Ileorektostomie oder als Diskontinuitätsresektion nach Hartmann mit endständigem Ileostoma durchgeführt wird.

\section{Frage: Wie sieht Ihr Entscheidungskonzept bei der oberen gastrointestinalen Blutung aus?}

Arbogast: Bei der endoskopisch gesicherten Quelle der oberen gastrointestinalen Blutung ist nach unserer Auffassung die erste Therapieoption die endoskopische lokale Therapie in Form von Clip-Applikationen, Unterspritzungen usw. Blutet es nach dem zweiten endoskopischen Blutstillungsversuch weiter, so ist in unserem Hause die Indikation zum operativen Vorgehen gegeben. Strengstens abgelehnt werden muss die immer wieder geäußerte Aussage, dass ein Patient mit nicht stillbarer Blutung zunächst mehrere Erythrozytenkonzentrate erhalten soll, um ihn in eine bessere Situation für die Operation zu bringen. Hier steht die kausale Therapie, d.h. die operative Blutstillung, an erster Stelle, da sich mit jeder transfundierten Konserve bekanntermaßen das Outcome der Patienten verschlechtert. Möglicherweise bieten die kombinierten Verfahren wie Laparoskopie und simultane Endoskopie hier vernünftige Therapieansätze. Eine letzte Würdigung dieser
Kombinationsverfahren kann jedoch zum gegenwärtigen Zeitpunkt noch nicht getroffen werden.

\section{Hagenmüller:}

1. Sicherung der Blutungsquelle durch Endoskopie.

2. Notfallendoskopie im Fall ungünstiger Prognosezeichen: hohes Lebensalter, Kreislaufinstabilität, Schock, Hämoglobin $<8 \mathrm{mg} / \mathrm{dl}$, anamnestisch-klinischer Verdacht auf Ösophagusvarizen als Quelle der Blutung.

3. Der endoskopischen Identifikation der Blutungsquelle folgt die endoskopische Blutstillung. Wenn diese erfolglos ist, Entscheidung für Operation.

4. Bei Rezidivblutung erneute Endoskopie gemeinsam mit dem chirurgischen Partner. In dieser Situation gemeinsame Einzelfallentscheidung über das weitere Blutstillungsvorgehen (erneute endoskopische Blutstillung oder Operation)

Izbicki: Das Entscheidungskonzept bei der Behandlung der oberen gastrointestinalen Blutung ist vorrangig davon abhängig, ob man über eine allzeit einsetzbare und versierte Endoskopie verfügt. Bei Zeichen einer frischen oder älteren stattgehabten Blutung im Magensaftsekret stellen wir die Indikation zur sofortigen Endoskopie. Sollte sich keine akute frische Blutung ergeben, so wird eine Therapie mit Protonenpumpenhemmern eingeleitet und innerhalb von 24-48 h erneut gastroskopiert. Sollte sich während dieses Zeitraumes ein relevanter Hämoglobinabfall zeigen, so wird die Endoskopiekontrolle vorverlegt. Die Blutstillung einer Forrest-Ia-Blutung sollte entweder durch Hämoclip-Applikation und/oder Suprarenininjektion bzw. wahlweise Fibrinunterspritzung erfolgen. Bei einem Transfusionsbedarf von $>6$ Konserven/24 h trotz endoskopischer Therapie sollte die Indikation zur Laparotomie sehr großzügig gestellt werden.

Rückert: Die obere gastrointestinale Blutung aus peptischen Ulzerationen oder anderen Läsionen als die Varizenblutung steht im Allgemeinen spontan. Anderenfalls sind eine aggressive Diagnostik und Therapie notwendig. Alle Patienten werden notfallmäßig endoskopiert, und bei $85-90 \%$ der Patienten lässt sich die Blutungsquelle identifizieren und nach Forrest klassifizieren. Die endoskopische Therapie führt zur Blutungskontrolle bei $>90 \%$ der blutenden Patienten. Wesentlich ist die Beurteilung des Rezidivblutungsrisikos (etwa 20\%). Alle Patienten erhalten eine adäquate Therapie mit Protonenpumpenhemmern. Bei Ösophagusvarizen erfolgt eine Sklerosierungstherapie.

Die konservative Behandlung ist die Basistherapie. Bei anhaltender Blutung (über 4 Einheiten Blut) und fehlgeschlagener endoskopischer Blutstillung erfolgt die Notfalloperation. Kommt es trotz erfolgreicher initialer endoskopischer Blutstillung zur Rezidivblutung (bis zu 20\%), ist ebenfalls die Operation indiziert. Im Forrest-Stadium Ia und Ib wird die Operation im frühen blutungsfreien Intervall angestrebt. Bei Forrest 
IIa mit sichtbarem Gefäßstumpf erfolgt ebenfalls die frühelektive Operation. Die Operationsletalität der Rezidivblutung des alten Menschen ist besonders hoch, so dass hier gerade die frühzeitige prophylaktische Operation nach initialer endoskopischer Blutstillung indiziert ist. Die Operation besteht vorzugsweise in der Kontrolle der Blutungsquelle mit intraluminärer Umstechung und gegebenenfalls extraluminärer Unterbindung von Gefäßen. Resezierende Verfahren und Vagotomie mit Drainage stellen die Ausnahme dar.

Weiser: Bei anamnestisch, klinisch und laborchemisch nachgewiesener oberer intestinaler Blutung erfolgt je nach zu erwartender Blutungsaktivität (Klassifikation nach Forrest-Kriterien) die Ösophagogastroduodenoskopie (ÖGD) mit dem Ziel der Blutungslokalisation, der Bestimmung der Blutungsaktivität und gegebenenfalls der endoskopischen Blutstillung, wobei als prognostisch ungünstige Kriterien bei der akuten Blutung des oberen Gastrointestinaltrakts sowohl für das endoskopische wie auch für das operative Procedere ein initialer $\mathrm{Hb}$-Wert von $<6 \mathrm{~g} / \mathrm{dl}$, initialer Konservenverbrauch $>6$ Einheiten/24 h, Lebensalter $>60$ Jahre, Multimorbidität und kurzfristig auftretende Rezidivblutungen gelten.

Wann Endoskopie: (1) In der Schocksituation sofortige Endoskopie unter Intubationsbedingungen mit dem Ziel der schnellstmöglichen Blutstillung bei zu erwartender hoher Blutungsaktivität. (2) Kein Schock, frische Hämatemesis: Baldmögliche Endoskopie, spätestens aber innerhalb von $6 \mathrm{~h}$ in Stand-by-Anästhesie. Da Blutungsintensität und -aktivität in der Regel nicht sicher abzuschätzen sind, ist das Ziel der ÖGD neben der Blutungslokalisation und -beurteilung ebenfalls die schnellstmögliche endoskopische Blutstillung. Auch bei Zeichen einer stattgehabten Blutung im Sinne sichtbarer Koagel oder Gefäßstümpfe erfolgt die prophylaktische Sklerosierung zur Vermeidung eines möglichen Blutungsrezidivs. (3) Kein Schock, alte Meläna, Hämoglobin 12 g/dl: Keine Notfallendoskopie, endoskopische Beurteilung der Situation innerhalb von $12 \mathrm{~h}$. Bei der hier zu erwartenden niedrigen Blutungsintensität und -aktivität und daraus resultierendem niedrigem Risiko sind Lokalisation der Blutungsquelle und Festlegung weiterer Therapiemaßnahmen innerhalb eines 12Stunden-Intervalls vertretbar.

Im Rahmen der ÖGD bzw. nach erfolgter endoskopischer Blutstillung werden Patienten mit endoskopischen Zeichen der Forrest-I- bzw. -II-Blutung ausnahmslos einer Intensivüberwachung zugeführt und mit zentralem Zugang bzw. Magensonde versorgt. Alle anderen Patienten benötigen nur eine Kreislaufüberwachung für etwa 48 h, da das Risiko einer Rezidivblutung gering ist.

Das Ergebnis der primären Endoskopie bestimmt das weitere Vorgehen: (1) Gelingt keine endoskopische Blutstillung, erfolgen unverzüglich die Laparotomie und chirurgische Blutstillung. (2) Gelingt die endoskopische Blutstillung, wird der Patient auf der Intensivstation bei liegender Magensonde unter hochdosierter konservativer Therapie überwacht. Pa- tienten mit hoher Blutungsintensität und -aktivität, z.B. bei Ulcus duodeni der Hinterwand oder einem penetrierenden Ulcus ventriculi an der kleinen Kurvatur (d.h. Ulzera im Einzugsbereich großer arterieller Gefäße oder Befunde mit einer Rezidivblutungsgefahr von um die $30 \%$ ) werden innerhalb von $6-8 \mathrm{~h}$ zur Kontrolle, gegebenenfalls zur neuerlichen endoskopischen Intervention reendoskopiert. Weniger gefährdete Patienten mit präpylorischen Ulzera, d.h. mit einem Rezidivblutungsrisiko zwischen $8-10 \%$, werden in 12 -stündigen Intervallen reendoskopiert. Bei Anzeichen einer Rezidivblutung nach 3-maligem endoskopischen Therapieversuch erfolgt die elektive Operation zur definitiven Blutstillung. Eine sofortige operative Blutstillung wird nur bei massiver, endoskopisch nicht beherrschbarer oberer instestinaler Blutung durchgeführt. Ausgenommen davon sind konservativ nicht stillbare Blutungen aus Ösophagusvarizen. In diesen Fällen bevorzugen wir zunächst die Ballontamponade mit späterer Einlage eines Tips. Eine Ausnahme stellen Einzelfälle mit blutenden Fundusvarizen dar, die als Ultima Ratio einer Sperroperation zugeführt werden können.

Kommentar: Die obere gastrointestinale Blutung stellt ein klassisches Krankheitsbild zur interdisziplinären Zusammenarbeit dar. Bei den Zeichen der Blutung erfolgt in jedem Fall die Endoskopie, die in Abhängigkeit von Kreislaufzustand und Komorbidität des Patienten entweder als Notfall sofort oder zum Wahlzeitpunkt durchgeführt wird. In einem großen Prozentsatz der Fälle gelingt es, durch endoskopische Maßnahmen eine Hämostase herbeizuführen. Kann die Blutung nicht primär gestillt werden, besteht die Oparationsindikation. Problematisch bleibt die Rezidivblutung, die in jedem Fall reendoskopiert werden sollte. Besteht weiterhin ein Bedarf an Blutkonserven zwischen 4 und 6 Erythrozytenkonzentraten pro $24 \mathrm{~h}$, so sollte großzügig die Operationsindikation gestellt werden.

\section{Frage: Akute nekrotisierende Pankreatitis: wie lange konservativ und wann operativ therapieren?}

Arbogast: Bei keinem Krankheitsbild ist die Zusammenarbeit zwischen Gastroenterologen und Viszeralchirurgen in so hohem Maße gefordert wie bei der akuten nekrotisierenden Pankreatitis. Von Anfang an, d.h. nach der Aufnahme in die Klinik, müssen die Patienten von Ärzten beider Fachgebiete regelmäßig gesehen und das weitere Procedere muss festgelegt werden. Nach unserer Auffassung kommt der Punktionsdiagnostik ein hoher Stellenwert zu, jedoch ist der entscheidende Faktor für ein letztlich operatives Vorgehen stets das Allgemeinbefinden des Patienten. Verschlechtert sich der Allgemeinzustand des Patienten mit nekrotisierender Pankreatitis so dramatisch, dass es zu einer respiratorischen und renalen Insuffizienz mit begleitender Sepsis führt, so ist das operative Vorgehen zwingend geboten, wenngleich eingeräumt wer- 
den muss, dass auch nach operativem Vorgehen die Letalitätsquote bei diesen Patienten extrem hoch ist. In unserer Klinik hat sich in diesen Fällen die programmierte Lavage mit vorsichtiger Nekrosektomie bewährt. Gleichwohl bleibt die genaue Festlegung des Zeitpunkts für das operative Vorgehen letztlich unklar und nur die Erfahrung stellt einen wesentlichen Parameter dar.

Hagenmüller: Konservative Therapie maximal ausreizen; bei Hinweis auf eine nekrotisierende Verlaufsform und/oder bakterielle Kontamination antibiotische Breitspektrumbehandlung. Bei zweifelhaftem Ansprechen der antibiotischen Behandlung sollte die kulturelle und serologische Suche nach einer Pilzinfektion erfolgen, die dann intensiv antibiotisch behandelt werden muss. Pilzinfektionen wurden in der Vergangenheit möglicherweise bei diesem Krankheitsbild zu wenig beachtet. Bei Organversagen intensivmedizinische Behandlung.

Zunehmende Bedeutung gewinnt die Möglichkeit der minimal invasiven Nekrosektomie, die offenbar in einer ganzen Reihe von Fällen zur Vermeidung der Laparotomie beitragen kann.

Izbicki: Prinzipiell sollte zunächst ein streng konservatives Vorgehen eingehalten werden. Indikationen zur Operation bestehen unseres Erachtens nur dann, wenn es unter konservativer Therapie zu einem dramatischen Fortschreiten des Pankreatitis-assoziierten Organversagens kommt oder wenn sich ein Anzeichen für eine infizierte Nekrose (beispielsweise Erregernachweis im Punktat) ergibt. Ebenso sollte die Indikation zur Operation erfolgen, wenn sich klinisch das Bild eines Kompartmentsyndroms zeigt.

Rückert: Die akute nekrotisierende Pankreatitis hat immer noch eine hohe Morbidität und Letalität. Aufgrund der zu Beginn durchgeführten Anamnese, von klinischer Untersuchung, Laborwerten, Bolusspiral-CT und selten ERCP erfolgt die Festlegung des Schweregrads nach einem Scoring-System (z.B. Schönborn-Kümmerle oder Ranson). Alle Patienten mit nekrotisierender Form der akuten Pankreatitis werden grundsätzlich zunächst konservativ intensivmedizinisch behandelt. Die aggressive Intensivmedizin führt in der Regel zu einer Kontrolle des Krankheitsbildes.

Bei fortschreitendem septisch-toxischem Schock und zunehmenden Organkomplikationen (Lunge, Niere) und/oder Nachweis der bakteriellen Infektion durch Nekrosenpunktion wird die Indikation zur Nekrosektomie gestellt. Vorzugsweise erst am Ende der 2. Woche nach Krankheitsbeginn und/oder Nachweis der bakteriellen Infektion der Nekrosen mit fortschreitender Entwicklung von Nekrosenstraßen im KontrollCT erfolgt die dann notwendige Operation. Das Timing ist essentiell, d.h. rechtzeitig vor Entwicklung irreversibler Organkomplikationen. Ziel der Operation ist die digitoklastische Entfernung von Nekrosen, die sich meist am Ende der
2. Woche so demarkiert haben, dass sie ohne große Blutungsoder Perforationsgefahr abgeräumt werden können. Resezierende Eingriffe sind in der Regel nicht angezeigt. Ein Laparostoma, ein Etappenlavage-Konzept und eine fallweise notwendige Kontrolle von Perforationen sind notwendig.

Weiser: Neben interventionellen oder chirurgischen Maßnahmen führen wir bei allen nekrotisierenden Verlaufsformen der akuten Pankreatitis eine Antibiotikatherapie mit nekrosegängigen Präparaten, z.B. Ofloxacin, durch. Lediglich in Einzelfällen wird nach Antibiogramm und Resistenztestung testgerecht umgestellt. Für die Dauer der Antibiotikatherapie lassen wir uns durch das klinische und laborchemische Bild leiten, die Applikation wird jedoch mindestens 10 Tage lang durchgeführt. Eine primäre antimykotische Prophylaxe bzw. die selektive Darmdekontamination erfolgt im eigenen Haus nicht. Die primäre intensivmedizinische Therapie hat die Behandlung der Sepsis, gegebenenfalls eines SIRS oder Multiorganversagens zum Ziel. Bei schweren Verlaufsformen mit respiratorischer Insuffizienz wird eine frühzeitige drucklimitierte Beatmung mit einem initialen PEEP von 5-10 mbar durchgeführt. Bei therapierefraktärem Anstieg der Retentionswerte oder unzureichend therapierbarer Hyperkaliämie erfolgt die frühzeitige Nierenersatztherapie mittels kontinuierlicher venöser Hämofiltration.

Da gesicherte Kriterien zur Annahme einer Nekroseninfektion nicht existieren und prophylaktische, ultraschallgezielte Feinnadelpunktionen auch zur Staphylococcus-Superinfektion führen können, erfolgt die Verlaufsbeobachtung über Klinik, Laborparameter, Sonographie- und CT-Kontrollen. In der Zusammenschau aller Kriterien kommt allerdings dem Kontrastmittel-CT für die Diagnose einer Nekroseninfektion wegweisende Bedeutung zu. Die Befunde des Kontrastmittel-CT werden durch Laborparameter wie z.B. Procalcitonin gestützt. Lediglich bei Verdacht auf eine Nekroseninfektion wird die gezielte, in der Regel ultraschallgestützte Feinnadelaspiration vorgenommen. Bei Vorliegen von Gaseinschlüssen im Kontrastmittel-CT, die für eine Infektion beweisend sind, wird auf die Durchführung der Feinnadelaspiration verzichtet.

Erst bei sicherem Nachweis infizierter peripankreatischer Nekrosen mit begleitender Verschlechterung der Vitalparameter wird die Indikation zur operativen Nekrosektomie gestellt. Wir bevorzugen die offene digitale Nekrosektomie mit anschließender Kompartimentbildung zur weiteren offenen Nekrosektomie und Lavage-Behandlung. Auf ausgedehnte Resektionen des Pankreas wird verzichtet, da nachwachsende Nekrosen über das Rechts- bzw. Linkslaparostoma digital mobilisiert und per Lavage effektiv entfernt werden können.

Lediglich beim Nachweis isolierter infizierter Pseudozysten oder abgedeckelter Pankreasabszesse wird primär interventionell therapiert, das Gleiche gilt für entzündungs- oder kompressionsbedingte Gallengangsobstruktionen.

Kommentar: Die Behandlung der akuten nekrotisierenden Pankreatitis erfolgt in enger Kooperation zwischen Gastroen- 
terologen und Viszeralchirurgen. Operationsindikationen sind Infektion der Nekrosen und zunehmende Sepsis und Multiorganversagen unter intensivmedizinischer Behandlung. Auch das Bild des abdominalen Kompartmentsyndroms kann die
Laparotomie und Druckentlastung erforderlich machen. Die operativen Maßnahmen bestehen in der Ausräumung der Nekrosen und der Kompartimentbildung. Die Behandlung erfolgt überwiegend in offenen Verfahren.

\section{Teilnehmer}

Prof. Dr. R. Arbogast

Städtisches Klinikum

Chirurgische Klinik

Postfach 1680

D-75116 Pforzheim (Deutschland)

Prof. Dr. F. Hagenmüller

1. Medizinische Abteilung

Allgemeines Krankenhaus Altona

Paul-Ehrlich-Straße 1

22763 Hamburg (Deutschland)
Prof. Dr. J.R. Izbicki

Klinik und Poliklinik für Chirurgie

Universitätsklinikum Hamburg-Eppendorf

Martinistraße 52

D-20246 Hamburg (Deutschland)

Prof. Dr. K. Rückert

Abteilung für Chirurgie

Klinikum Nord

Tangstedter Landstraße 400

D-22417 Hamburg (Deutschland)
Prof. Dr. H.-F. Weiser

I. Chirurgie

Diakoniekrankenhaus

Postfach 1211

D-27342 Rotenburg/Wümme (Deutschland) 